\title{
A case of follow-up of a patient with 22q11.2 distal deletion syndrome and a review of the literature
}

\author{
Dong Jun $\mathrm{Ha}^{1 \oplus}$, Ji Sun Park ${ }^{1,2 \oplus}$, Woori Jang ${ }^{2,3 \oplus}$, Na-young Jung ${ }^{1 \oplus}$, Su Jin Kim ${ }^{1,2}$, Yeonsook Moon ${ }^{2,3 \oplus}$, and Jieun Lee ${ }^{1,2, *}$ \\ ${ }^{1}$ Department of Pediatrics, Inha University Hospital, Inha University College of Medicine, Incheon, Korea \\ ${ }^{2}$ Northwest Gyeonggi Regional Center for Rare Disease, Incheon, Korea \\ ${ }^{3}$ Laboratory Medicine, Inha University Hospital, Inha University College of Medicine, Incheon, Korea
}

Microdeletions of chromosome 22q11.2 are one of the most common microdeletions occurring in humans, and is known to be associated with a wide range of highly variable features. These deletions occur within a cluster of low copy repeats (LCRs) in 22q11.2, referred to as LCR22 A-H. DiGeorge (DGS)/velocardiofacial syndrome is the most prevalent form of a $22 q 11.2$ deletions, caused by mainly proximal deletions between LCR22 A and D. As deletions of distal portion to the DGS deleted regions has been extensively studied, the recurrent distal 22q11.2 microdeletions distinct from DGS has been suggested as several clinical entities according to the various in size and position of the deletions on LCRs. We report a case of long-term follow-up of a female diagnosed with a 22q11.2 distal deletion syndrome, identified a deletion of $1.9 \mathrm{Mb}$ at 22q11.21q11.23 (chr22: 21,798,906-23,653,963) using single nucleotide polymorphism array. This region was categorized as distal deletion type of 22q11.2, involving LCR22 D-F. She was born as a preterm, low birth weight to healthy non-consanguineous Korean parents. She showed developmental delay, growth retardation, dysmorphic facial features, and mild skeletal deformities. The patient underwent a growth hormone administration due to growth impairment without catch-up growth. While a height gain was noted, she had become overweight and was subsequently diagnosed with pre-diabetes. Our case could help broaden the genetic and clinical spectrum of 22q11.2 distal deletions.

Key words: Chromosome 22q11.2 deletion syndrome, Distal, Microarray analysis, Mitogen-activated protein kinase 1, Short stature, Obesity.

\section{Introduction}

Microdeletions of chromosome 22q11.2 (22q11.2 deletion syndrom, DS) are the most common microdeletions and affect around 1 in 4,000 live births. 22q11.2DS occurs due to a heterozygous deletion medicated by meiotic chromosomal rearrangements. In humans, the proximal long arm of chromosomes 22 has the greatest proportion of low-copy repeats (LCRs), referred to as LCR22 A-H [1]. LCRs are known as segmental duplications and highly homogenous sequences of genome, which can mediate recurrent copy number variants (CNVs) such as microdeletion or microinversions [2]. DiGeorge (DGS)/velocardiofacial syndrome is the most prevalent form of a 22q11.2DS, caused by proximal deletions in 22q11.2 which are two types with LCR22 $A-D$, and A-B. As other deletions of distal portion to the DGS deleted regions has been extensively studied, 22q11.2DS can be

\footnotetext{
Received: 7 October 2021, Revised: 8 November 2021, Accepted: 8 November 2021, Published: 31 December 2021

*Corresponding author: Jieun Lee, M.D., Ph.D. (iD https://orcid.org/0000-0002-7386-0015

Department of Pediatrics, Inha University Hospital, Inha University College of Medicine, 27 Inhang-ro, Jung-gu, Incheon 22332, Korea.

Tel: +82-32-890-3579, Fax: +82-32-890-2844, E-mail: anicca@inha.ac.kr

Conflict of interest: The authors declare that they do not have any conflicts of interest.
}

(c) This is an open-access article distributed under the terms of the Creative Commons Attribution Non-Commercial License (http://creativecommons.org/licenses/by-nc/4.0/) which permits unrestricted non-commercial use, distribution, and reproduction in any medium, provided the original work is properly cited.

(c) Copyright 2021 by the Korean Society of Medical Genetics and Genomics 
categorized into proximal, central, and distal deletions according to the region and extent of the rearrangements associated with the LCRs (Fig. 1) [3,4]. Proximal deletion including the DGS critical genes HIRA or $T B X 1$, is characterized by congenital heart defects (CHDs), velopharyngeal insufficiency, immunodeficiency, hypocalcemia, hypotonia, intellectual disability (ID) and/ or behavioral problems, and growth restriction [5]. Central deletions span either B-D or C-D, located/nested at the distal end of the DGS interval. The characteristics of the central deletion are similar to those of the proximal deletion, but the incidence of hypotonia and immunodeficiency is much lower than that of the proximal deletion. Distal deletions are classified as type I, II, and III. Characteristics of distal deletions are that, although hypocalcemia seen in proximal deletions is rare, skeletal abnormalities and cardiovascular defects are more frequent than central deletions [3].

The previous diagnosis of 22q11.2DS was performed through the fluorescence in situ hybridization. Due to diagnostic limitations and phenotypic variability, it seems that 22q11.2 distal DS can be "silent" and it is unknown how many people may have a silent form of this syndrome. Since the recent introduction of health insurance coverage for the chromosomal microarray analysis (CMA) tests in Korea, CMA tests have been broadly used to detect the exact location and size of CNVs in individuals with unexplained developmental delay (DD)/ID, autism spectrum disorders or multiple congenital anomalies [6]. Undiagnosed $22 q 11.2$ distal DS patients with DD/ID or multiple congenital anomalies are expected to be diagnosed more in the future. Thus we report a case of long-term follow-up of a patient diagnosed with 22q11.2 distal DS using CMA test and a review of the literature. Written informed consents and permission to use the images were obtained from her parents.

\section{Case}

\section{Clinical features}

A 3-year-old female was referred to our hospital for the evaluation of global DD and growth retardation. She was born at preterm, 35 weeks and 2 days of gestation after a normal pregnancy to healthy non-consanguineous Korean parents. The patient had two healthy and non-obese siblings with no significant family history. The birth weight of the patient was 1,830 $\mathrm{g}$ (4th percentile) and head circumference was $29.5 \mathrm{~cm}$ (4th percentile). Neonatal neurosonography revealed no abnormal finding excluding mild germinal matrix hemorrhage.

At the 3-year-old age, she presented global DD with speech delay, and growth retardation such as both height and weight below the $3 \mathrm{rd}$ percentiles $(84.7 \mathrm{~cm}$ and $10.2 \mathrm{~kg}$ ) for age and sex. The laboratory result of inborn error of metabolism was within normal range. Conventional chromosomal analysis and fragile X syndrome test showed no abnormal results. Her thyroid function test and other biochemical findings were all normal. Developmentally, the female had mild delays in achieving motor and cognitive mile stones, but significant delays in the language area (12-month level).

At the age of 5 , her growth was still retarded with a height of
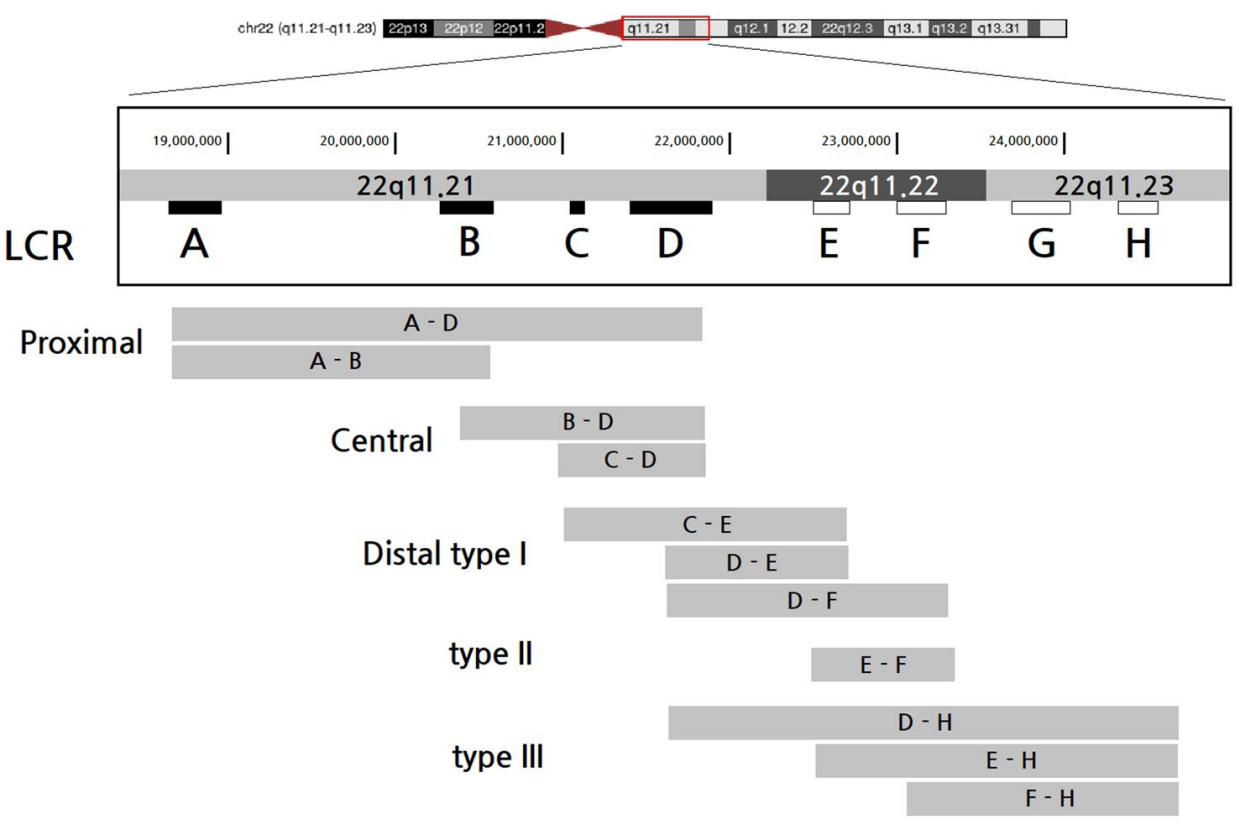

Fig. 1. Schematic diagram expressing the 22q11.2 region and LCR22 A-H contained therein. LCR, low copy repeat. Revised from the article of Burnside RD (Cytogenet Genome Res 2015;146:89-99) [3] and UCSC Genome Browser on Human Feb. 2009 (GRCh37/hg19) Assembly [4]. 
$92.3 \mathrm{~cm}(<3 \mathrm{rd}$ percentile) and a weight of $11.7 \mathrm{~kg}(<3 \mathrm{rd}$ percentile) respectively. Clinical evaluation revealed dysmorphic face including an inverted triangular shaped face, low set ears, high nasal bridge, smooth philtrum, thin upper lip. In addition to mild scoliosis due to leg length discrepancy, 5th finger clinodactyly and pes planus on both feet were present (Fig. 2). The bone age was delayed 2 years compared to the chronological age. Brain magnetic resonance imaging performed showed no structural abnormalities. In the L-dopa stimulation test and the insulin stimulation test, the growth hormone $(\mathrm{GH})$ peak was measured to be 2.34 and $6.88 \mathrm{ng} / \mathrm{mL}$, respectively, and the patient under- went a GH administration due to no postnatal catch-up growth with subnormal GH secretory response to two provocative $\mathrm{GH}$ testing.

After 31 months of $\mathrm{GH}$ administration, the patient's height gradually increased to about the 3rd percentile, but the body weight sharply increased to the 50th percentile for age and gender. Truncal obesity developed gradually (Fig. 3). GH administration was discontinued to prevent the acceleration of gait disturbance due to both feet pain. Her full-scale intelligence quotient (FSIO) at the age of 8 using the Korean Wechsler Intelligence Scale for Children-III was 51, indicating mild ID. The girl

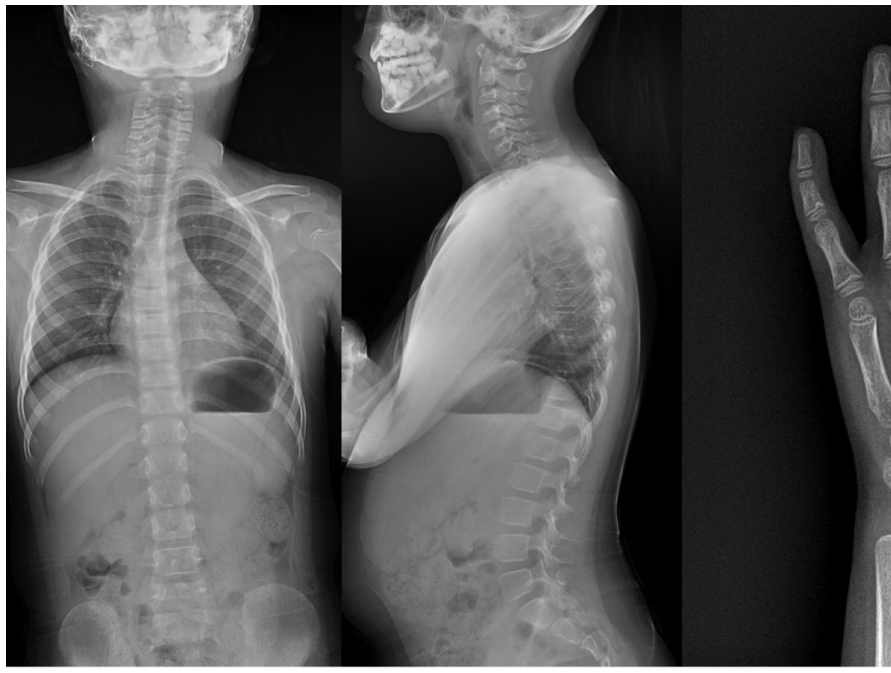

A

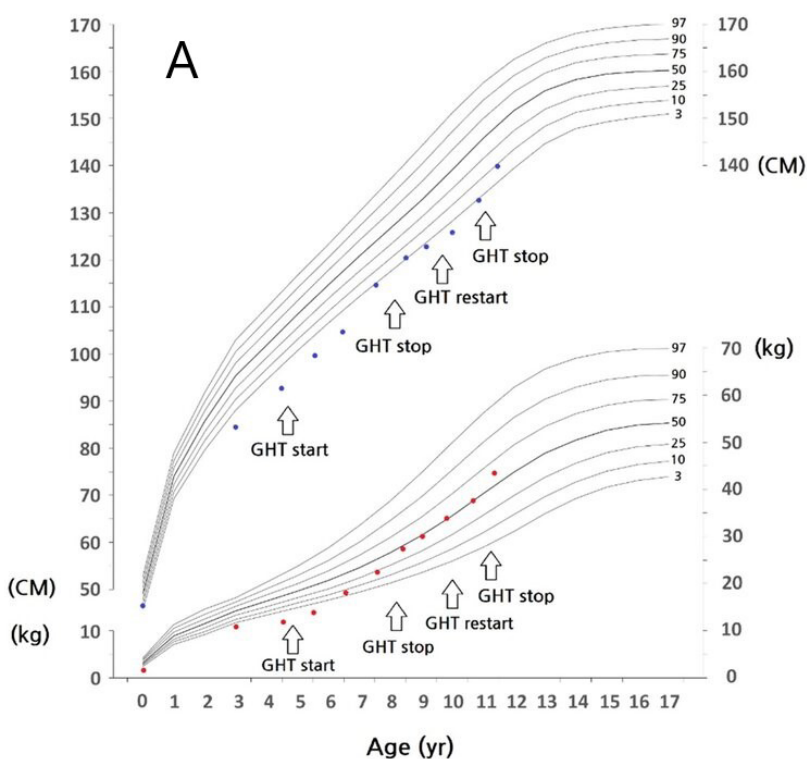

Fig. 2. A thoracolumbar spine and hand $X$-ray of 8-year-old patient showed mild scoliosis (A) and 5th finger clinodatyly (B), respectively.

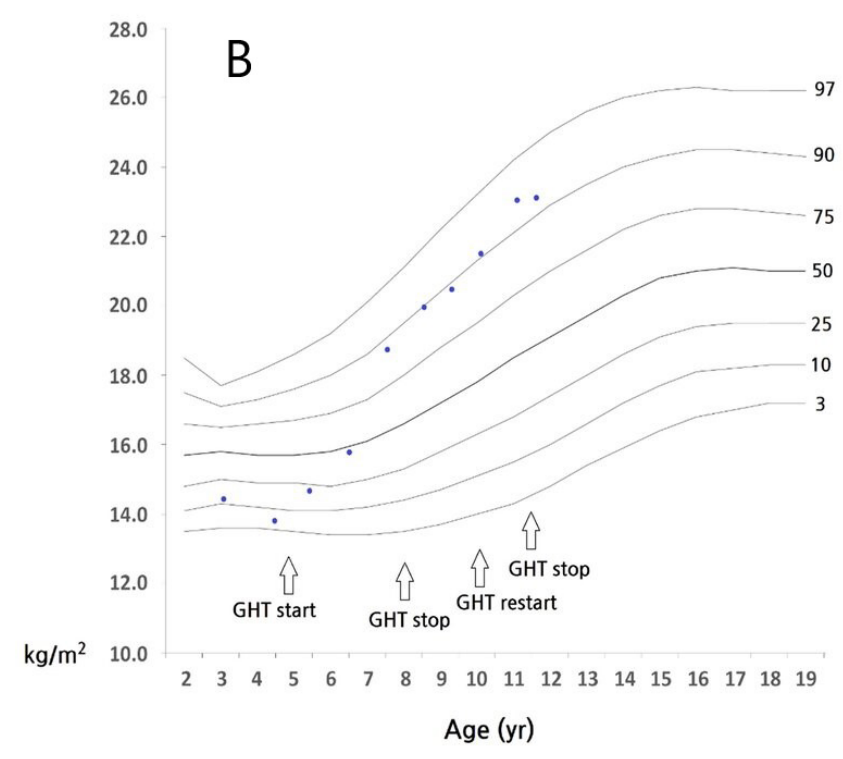

Fig. 3. Growth chart of the patient under GHT. (A) Upper side shows height chart and lower side shows weight chart. The dots indicate the height and weight measurement. The arrow marks the beginning or end of GHT. The patient's height velocity after growth hormone administration was increased $8 \mathrm{~cm} / \mathrm{yr}$. (B) It shows the change in body mass index according to the age of the patient. GHT, recombinant human growth hormone therapy. 


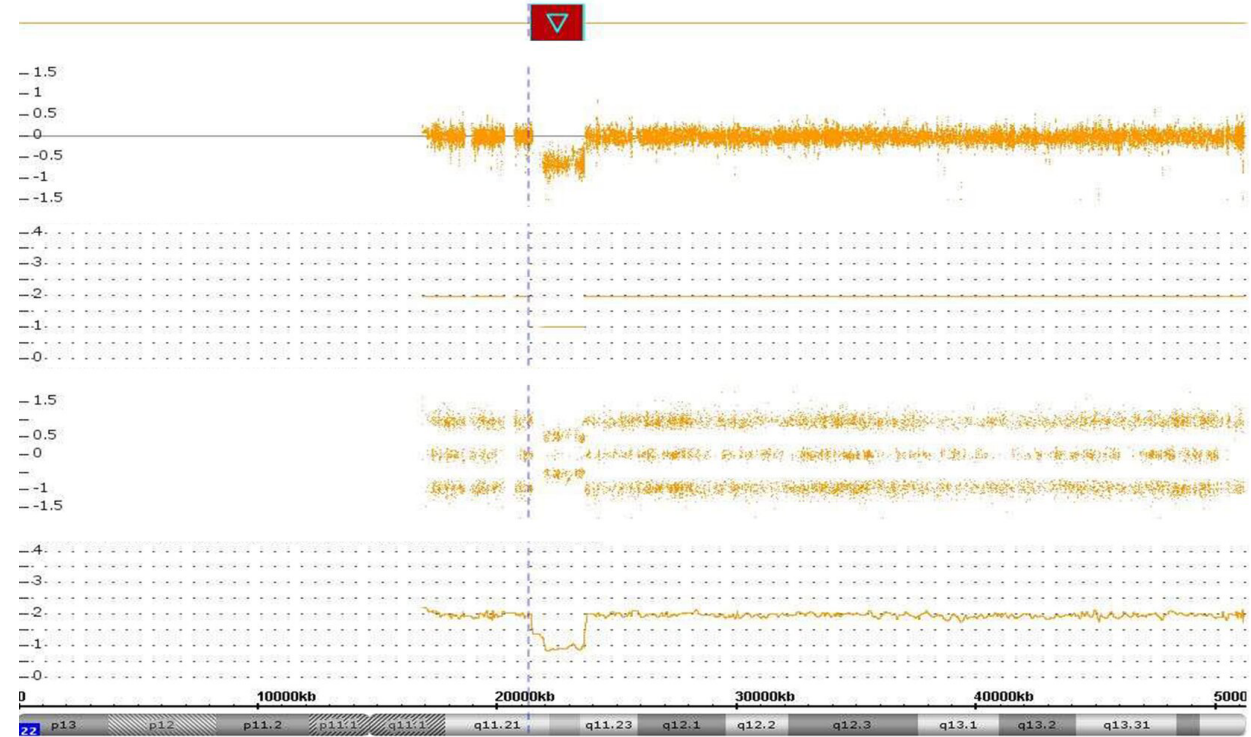

Fig. 4. Chromosomal microarray analysis image showing a $1.9-\mathrm{Mb}$ deletion at 22q11.21q11.23 (chr22: 21,798,906$23,653,963)$, which was classified as likely pathogenic copy number variants. had poor performance in learning and social adaptation skill.

At 10 year of age, she was received $\mathrm{GH}$ therapy again due to deceleration of height velocity ( $<3$ rd percentile). At the age of 11, while her sexual maturity ratings were Tanner stage II for breast, and Tanner I stages for pubic and axillary hair. Her bone age was about 2 years older than her chronological age. Her body mass index Z-score was 1.97 (97th percentile), which was overweight (Fig. 3). Although there was no evidence of acanthosis nigricans, she also was diagnosed as having pre-diabetes with an $\mathrm{HbA} 1 \mathrm{C}$ level of $6.2 \%$ and mild fatty liver proven by an abdominal ultrasound. However, she did not show dyslipidemia on lipid pannel. On the social maturity scale, her developmental status was scored as 6 years for social age and the social quotient was 62 . She had an FSIO of 54 at the age of 11, implicating mild ID. CMA testing revealed chromosome 22q11.2 distal microdeletion of the patient. Echocardiogram, renal ultrasound, immunologic and auditory assessments were all normal.

\section{Genetic analysis}

CMA was performed with CytoScan ${ }^{\mathrm{TM}}$ Dx Assay (Thermo Fisher Scientific., Santa Clara, CA, USA) on genomic DNA from peripheral blood leukocytes of the patient. Array data was analyzed using the Chromosome Analysis Suite Dx software (Thermo Fisher Scientific). Human Genome Build 19 was used to perform the description of genomic variants.

A heterozygous deletion of maximum 1.9-Mb spanning from $22 q 11.21$ to $22 q 11.23$ was identified: $\operatorname{arr}$ [GRCh37] $22 q 11.21 q 11.23(21,798,906-23,653,963) \times 1$, which had likely pathogenic significance (Fig. 4). This deleted region contained
16 Online Mendelian Inheritance in Man genes including $H / C 2$, UBE2L3, SDF2L1, MIR 13OB, PPIL2, YPEL1, MAPK1, TOP3B, VPREB1, PRAME, GGTLC2, MIR650, RTDR1, GNAZ, RAB36, and $B C R$.

\section{Discussion}

In this case study, we describe the clinical features of a girl with 22q11.2 distal DS from neonate to school age through a long-term follow-up. Our patient showed mild ID, growth failure, mild skeletal abnormalities, and dysmorphic facial features, but no apparent facial dysmorphism, no hypocalcemia, no frequent infection or cardiac/palatal anomalies, which are typical features for DGS. The recurrent distal 22q11.2 microdeletions distinct from DGS have been suggested as several clinical entities according to the various in size and position of the deletions on LCRs. With the introduction of CMA tests, the diagnostic rate of central and distal deletions of 22q11.2 would be expected to improve in the near future. To date, this might be the first case report with the recurrent distal 21q11.2DS using CMA test in Korea so far.

According to the published reports, about half of the patients with distal deletions were type I. The frequency of short stature was $56 \%$, higher than that of other distal types. The proportion of microcephaly and skeletal anomalies is also high [3]. Dysmorphic features were observed over half of the patients with distal deletions, such as smooth philtrum, ear abnormalities, thin upper lip, upslanting palpebral fissures, and pointed chin. Our case girl showed similar clinical features. Although genetic testing of 


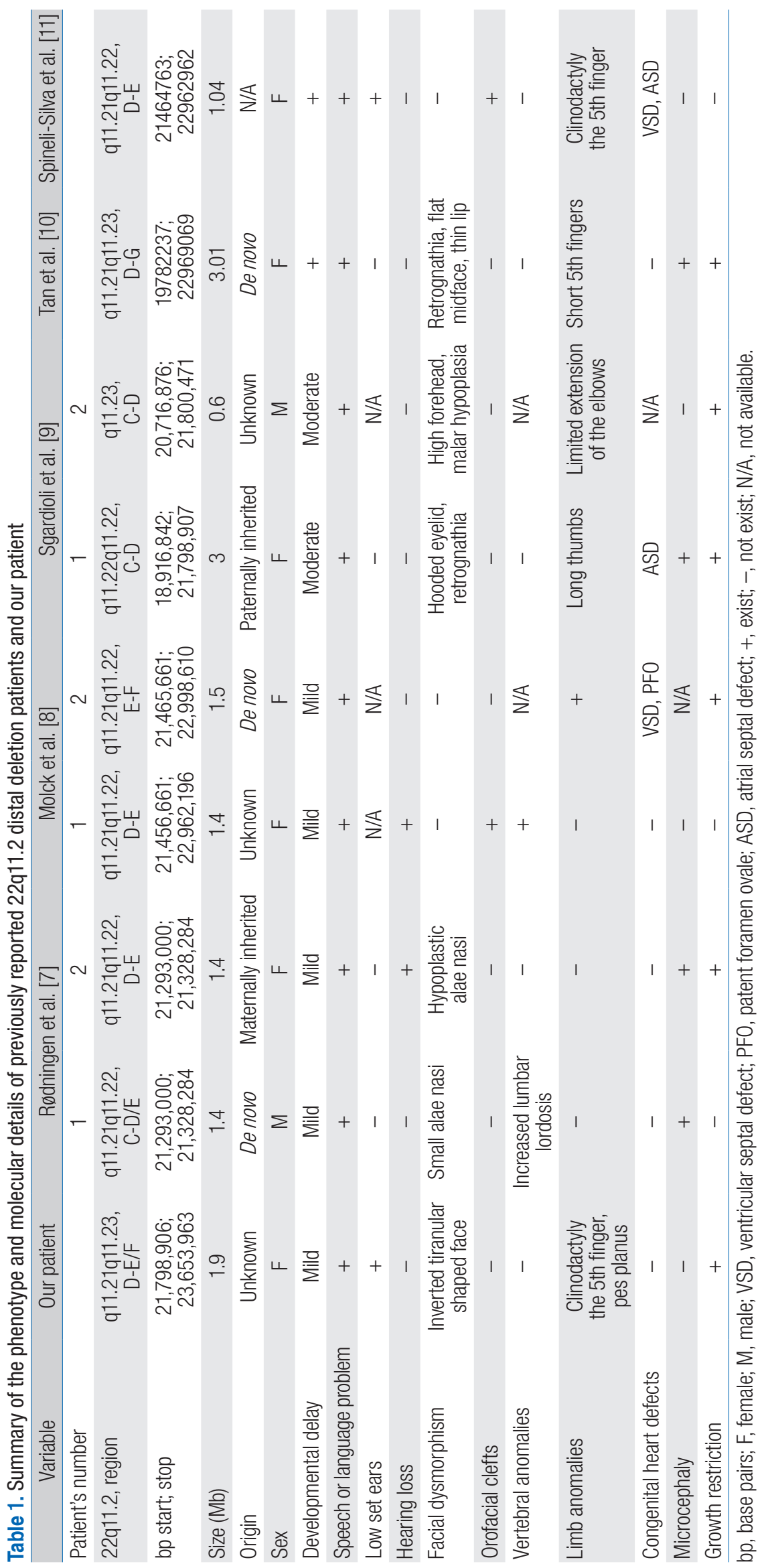


the other family members was not performed, the deletion of our patient could be estimated de novo because other all family members were non-affected. Most type I distal deletions are reported as de novo (62\%) similar to the high rate of de novo deletion for proximal deletion of 21q11.2DS [3]. Table 1 [7-11] is described summary of the phenotype and molecular details of previously reported 22q11.2 distal DS patients including our case.

Identified deletion of $1.9 \mathrm{Mb}$ at 22q11.21q11.23 (chr22: 21,798,906-23,653,963) of our case was spanning LCR22 D-F. This region can be categorized into distal type I deletion of 22q11.2, which is included distal CNVs [3]. Notably, pre and/or post growth restriction was noted in most patients (78\%) with either the LCR22 D to E deletion or the LCR22 D to F deletion [12]. Our case patient also was intrauterine growth retardation prenatally and small for gestational age at birth. This region LCR22 D-E had been suggested the critical region for growth retardation and included the MAPK 1 gene. MAPK 1 encodes mitogen-activated protein kinase 1 and has been proposed to be a candidate for the prematurity and/or low birth weight in distal $22 q 11.2$ microdeletions due to the role of involving in placental development [13].

Global DD with a prominent language delay and mild to moderate ID were noted in more than half of patients (50\%-80\%) with the type of distal deletions. All cases with the same deleted region share some phenotypic features, however there is clinical heterogeneity. Deletion of TOP $3 B$ have been associated with cognitive impairment and facial dysmorphism in a patient with 22q11.2DS [14].

Unlike our patient, some individuals ( $50 \%)$ with distal deletion type I were observed CHD such as septal defects. The candidate genes involved in CHD are MAPK 1, HICS, CRKL. Cardiac defects in these patients might be caused by position effect on the MAPK1 gene, haploinsufficiency of a gene localized in the deleted region, exposure of a recessive mutation on the remaining allele of a gene in the deleted region. Mouse models also suggest that MAPK $1 / 3$ signaling may be disrupted in the common $22 q 11 \mathrm{DS}$, and that this is caused by disturbance of the fgf8 signaling [15].

Our case consistently presented growth retardation below the 3rd percentile for age and gender during growing-up. Short stature is another common issue associated with the 22q11.2DS. Newborns with the 22q11.2DS are smaller than the normal population. Growth in the 22q11.2DS is slower in infancy and childhood According to studies, it was reported that the final adult height of 22q11.2DS is generally smaller than the general population, and the average height is 166 to $167.2 \mathrm{~cm}$ for men and 152 to $158.2 \mathrm{~cm}$ for women, respectively [16]. However, other studies have reported that the adolescent and final adult heights were usually in the normal range [17]. Although our case patient showed a subnormal GH secretory response to two provocative $\mathrm{GH}$ testing, average growth velocity of this girl was normal. The effect of $\mathrm{GH}$ treatment for this case was unclear. After GH therapy for several years, her height reached about the 10th percentile on the growth curve for normal population. Because our patient did not reach near adult height, it seems that there is potential for linear growth, but the final height is thought to be smaller than the median height of normal population because the bone age is advanced compared to the chronological age. Further studies on the relationship between 22q11.2DS and height are needed to predict the final height of patients with short stature and to make an early treatment plan.

One of the unique characteristics of this patient was developed obesity. There are several studies on the association between 22q11.2DS and obesity. A Canadian cohort study found that the proportion of obese adult (body mass index $>30$ ) with 22 q11.2DS was 43.5\%, and obesity with type 2 diabetes was associated with 22q11.2DS [18]. There is a tendency to develop obesity in adolescents with 22q11.2DS [19]. Obesity is resulted from bulimia. Obsessive behaviors secondary to some of the psychiatric features is commonly seen in 22q11.2DS that are similar to Prader-Willi Syndrome (PWS) [20]. Therefore, 22q11.2DS should be managed similarly to PWS. In order to prevent our patient from progressing to adult obesity and type 2 diabetes, not only life style modification but also psychiatric management should be accompanied.

In conclusion, our case could help broaden the genetic and clinical spectrum of 22q11.2 distal DS with the course of GH administration. In addition, we have shown the long-term clinical course and auxological changes in patients following $\mathrm{GH}$ treatment.

\section{Author's Contributions}

Conception and design: JL. Acquisition of data: SJK, JSP. Analysis and interpretation of data: DJH, NJ, YM. Drafting the article: DJH. Critical revision of the article: WJ. Final approval of the version to be published: all authors.

\section{References}

1. Bailey JA, Eichler EE. Primate segmental duplications: crucibles of evolution, diversity and disease. Nat Rev Genet 2006;7:552-64. 
2. Low copy repeats. In: Ganten D, Ruckpaul K, Birchmeier W, Epplen JT, Genser K, Gossen M, eds. Encyclopedic reference of genomics and proteomics in molecular medicine. Berlin-Heidelberg: Springer, 2006.

3. Burnside RD. 22q11.21 Deletion syndromes: a review of proximal, central, and distal deletions and their associated features. Cytogenet Genome Res 2015;146:89-99.

4. UCSC Genome Browser on Human Feb. 2009 (GRCh37/hg19) Assembly [Internet]. Santa Cruz, CA: University of California, Santa Cruz, 2009. [http://genome.ucsc.edu/cgi-bin/hgTracks?db=hg 19\&tlastVirt ModeType $=$ defaultctlastVirtModeExtraState $=\varepsilon$ tvirtModeType $=$ defa ultetvirtMode=0\&tnonVirtPosition=etposition=chr22\%3A1800000 0\%2D24500000\&hgsid=1148663159_n9kWeMIHchvJUxiUTExIASCYbbL1]

5. McDonald-McGinn DM, Kirschner R, Goldmuntz E, Sullivan K, Eicher P, Gerdes M, et al. The Philadelphia story: the 22q11.2 deletion: report on 250 patients. Genet Couns 1999;10:11-24.

6. Blue Cross Blue Shield Asssociation. Special report: chromosomal microarray for the genetic evaluation of patients with global developmental delay, intellectual disability, and autism spectrum disorder. Technol Eval Cent Assess Program Exec Summ 2015;30:1-4.

7. Rødningen $O K$, Prescott $T$, Eriksson $A S$, Røsby $0.1 .4 \mathrm{Mb}$ recurrent 22q11.2 distal deletion syndrome, two new cases expand the phenotype. Eur J Med Genet 2008;51:646-50.

8. Molck MC, Vieira TP, Sgardioli IC, Simioni M, Dos Santos AP, Souza J, et al. Atypical copy number abnormalities in 22q11.2 region: report of three cases. Eur J Med Genet 2013;56:515-20.

9. Sgardioli IC, de Mello Copelli M, Monteiro FP, Dos Santos AP, Lustosa Mendes E, Paiva Vieira T, et al. Diagnostic approach to microdeletion syndromes based on 22q11.2 investigation: challenges in four cases. Mol Syndromol 2017;8:244-52.

10. Tan TY, Collins A, James PA, McGillivray G, Stark Z, Gordon CT, et al. Phenotypic variability of distal 22q11.2 copy number abnormalities.
Am J Med Genet A 2011;155A:1623-33.

11. Spineli-Silva S, Bispo LM, Gil-da-Silva-Lopes VL, Vieira TP. Distal deletion at 22q11.2 as differential diagnosis in craniofacial microsomia: case report and literature review. Eur J Med Genet 2018;61:262-8.

12. Mikhail FM, Burnside RD, Rush B, Ibrahim J, Godshalk R, Rutledge SL, et al. The recurrent distal 22q11.2 microdeletions are often de novo and do not represent a single clinical entity: a proposed categorization system. Genet Med 2014;16:92-100.

13. Aouadi M, Binetruy B, Caron L, Le Marchand-Brustel Y, Bost F. Role of MAPKs in development and differentiation: lessons from knockout mice. Biochimie 2006;88:1091-8.

14. Kaufman CS, Genovese A, Butler MG. Deletion of TOP3B is associated with cognitive impairment and facial dysmorphism. Cytogenet Genome Res 2016;150:106-11.

15. Moon AM, Guris DL, Seo JH, Li L, Hammond J, Talbot A, et al. Crkl deficiency disrupts Fgf8 signaling in a mouse model of $22 q 11$ deletion syndromes. Dev Cell 2006;10:71-80.

16. Tarquinio $\mathrm{DC}$, Jones $\mathrm{MC}$, Jones $\mathrm{KL}$, Bird LM. Growth charts for $22 \mathrm{q} 11$ deletion syndrome. Am J Med Genet A 2012;158A:2672-81.

17. Ryan AK, Goodship JA, Wilson DI, Philip N, Levy A, Seidel H, et al. Spectrum of clinical features associated with interstitial chromosome 22q11 deletions: a European collaborative study. J Med Genet 1997;34:798-804

18. Voll SL, Boot E, Butcher NJ, Cooper S, Heung T, Chow EW, et al. Obesity in adults with 22 q11.2 deletion syndrome. Genet Med 2017;19:204-8.

19. Digilio MC, Marino B, Cappa M, Cambiaso P, Giannotti A, Dallapiccola B. Auxological evaluation in patients with DiGeorge/velocardiofacial syndrome (deletion 22q11.2 syndrome). Genet Med 2001;3:30-3.

20. Bassett JK, Chandler KE, Douzgou S. Two patients with chromosome 22q11.2 deletion presenting with childhood obesity and hyperphagia. Eur J Med Genet 2016;59:401-3. 